\title{
MAASS-JACOBI FORMS OVER COMPLEX QUADRATIC FIELDS
}

\author{
Kathrin Bringmann, Charles H. Conley, and Olav K. Richter \\ ABSTRACT. We use methods from representation theory and invariant theory to compute \\ differential operators invariant under the action of the Jacobi group over a complex \\ quadratic field. This allows us to introduce Maass-Jacobi forms over complex quadratic \\ fields, which are Jacobi forms that are also eigenfunctions of an invariant differential \\ operator. We present explicit examples via Jacobi-Eisenstein series.
}

\section{Introduction}

In 1949, Maass [15] systematically investigated non-analytic automorphic forms which are eigenfunctions of the non-Euclidean Laplace operator. This lead to the theory of Maass waveforms, which has since grown enormously and is intimately linked to the spectral theory of automorphic forms. For a good overview (including many references) of Maass waveforms, see for example Hejhal [9] and [10], Terras [23], Bump [2], Iwaniec [13], and Iwaniec and Kowalski [14].

Several generalization of Maass waveforms have been considered: Siegel-Maass forms (for example, see Maass [16]), Maass forms over complex quadratic fields (for example, see Elstrodt, Grunewald, and Mennicke [4]), and Maass-Jacobi forms over the rationals (see Berndt and Schmidt [1] and Yang [24]).

Skogman [21] and Richter and Skogman [19] use Jacobi theta functions to construct Jacobi forms over complex quadratic fields and arbitrary number fields, respectively. The classical Jacobi forms in Eichler and Zagier [3] are holomorphic functions and more generally, the Maass-Jacobi forms over the rationals in [1] and [24] are eigenfunctions of a differential operator invariant under the real Jacobi group. However, in the case of a complex quadratic field, it had not been known whether the Jacobi theta functions in [21] and [19] satisfy an analgous differential equation. In this work we give such a condition.

Our paper divides naturally into two parts: the first three sections concern number theory and the last two concern representation theory. In the first part we initiate a theory of Maass-Jacobi forms over complex quadratic fields. We use differential operators invariant under the slash actions of the Jacobi group considered in [21] and [19] to define Maass-Jacobi forms over complex quadratic fields. These forms are Jacobi forms over complex quadratic fields (as in [21] and [19]) which in addition are eigenfunctions of one of the invariant differential operators. This last condition is the analog of the eigenfunction condition for the Maass-Jacobi forms alluded to above. We present explicit non-trivial examples of such forms via Jacobi-Eisenstein series.

Received by the editors July 25, 2006.

2000 Mathematics Subject Classification. Primary 11F50; Secondary 43A85. 
The second part of the paper is dedicated to the computation of the invariant operators needed in the first. In Section 4 we review the general framework within which one computes differential operators invariant under a Lie group action. This material is well-known "folklore" in representation theory, but we do not know of any reference in which it is all drawn together. Some of it is given in Helgason [11] from a different point of view.

In Section 5 we define the complex Jacobi group $G$, a central extension of the complex version of the Jacobi group used in the number-theoretic part of the paper. (The central extension is necessary in the complex setting in order to make the slash actions into group actions.) We begin with two presentations of $G$, analogous to those in [3] and [1]. Then in Section 5.1 we give an efficient conceptual derivation of the formula for the action of $G$ on the space underlying the slash actions. In Section 5.2 we use invariant theory to obtain a complete description of the graded algebras of the algebras of slash-invariant differential operators.

In Section 5.3 we compute the required invariant differential operators, a very long and intricate calculation. We begin by verifying that our slash actions really are group actions, already a non-trivial computation, which to our knowledge is not yet in print. We conclude in Section 5.4 with some remarks and directions for further research. We also give the invariant operators in two alternate coordinate systems, complex S-coordinates (see [1]) and quaternionic coordinates.

\section{Notation and terminology}

Let $K$ be the complex quadratic field with discriminant $d_{K}$. The algebraic conjugate of an algebraic number $\alpha$ is identical with its complex conjugate and is denoted by $\bar{\alpha}$. Let $\mathfrak{d}_{K}$ be the different of $K$, let $\mathcal{O}_{K}$ be the ring of integers of $K$, and set $\Gamma:=\mathrm{SL}_{2}\left(\mathcal{O}_{K}\right)$. The Jacobi group of $K$ is $\Gamma^{J}(K):=\Gamma \ltimes \mathcal{O}_{K}^{2}$.

The full ring of quaternions $\mathcal{Q}$ and the quaternionic upper half space $\mathbb{H}_{\mathcal{Q}}$ are defined by

$$
\begin{aligned}
\mathcal{Q} & :=\left\{u+v \mathbf{k} \mid u, v \in \mathbb{C}, \mathbf{k}^{2}=-1, a \mathbf{k}=\mathbf{k} \bar{a}, \forall a \in \mathbb{C}\right\} \\
\mathbb{H}_{\mathcal{Q}} & :=\left\{x+y \mathbf{k} \in \mathcal{Q} \mid x \in \mathbb{C}, y \in \mathbb{R}^{+}\right\} .
\end{aligned}
$$

Recall the well-known action $\left(\begin{array}{cc}\alpha & \beta \\ \gamma & \delta\end{array}\right) \circ \tau:=(\alpha \tau+\beta)(\gamma \tau+\delta)^{-1}$ of $\Gamma$ on $\mathbb{H}_{\mathcal{Q}}$. It extends to the following action of $\Gamma^{J}(K)$ on $\mathbb{H}_{\mathcal{Q}} \times \mathcal{Q}$ :

$$
\left[\left(\begin{array}{ll}
\alpha & \beta \\
\gamma & \delta
\end{array}\right),(\lambda, \mu)\right] \circ(\tau, z):=\left(\left(\begin{array}{cc}
\alpha & \beta \\
\gamma & \delta
\end{array}\right) \circ \tau,(\tau \gamma+\delta)^{-1}(z+\tau \lambda+\mu)\right) .
$$

For $z=u+v \mathbf{k} \in \mathcal{Q}$, define $\bar{z}:=\bar{u}-v \mathbf{k}, \tilde{z}:=u+\bar{v} \mathbf{k}$, and $\mathcal{N}(z):=z \bar{z}=|u|^{2}+|v|^{2}$, the usual quaternion norm. We also define $\mathcal{T}_{\mathcal{R}}[z]:=\|z\|_{\mathbb{C}}+\|z\|_{\mathbb{C}}$, where $\|u+v \mathbf{k}\|_{\mathbb{C}}:=$ $u+i v$ and $\|u+v \mathbf{k}\|_{\overline{\mathbb{C}}}:=\bar{u}+i \bar{v}$.

Henceforth fix $k \in \mathbb{C}, l \in \mathbb{N}$, and $m \in \mathbb{C}^{l}$ such that ${ }^{t} m m \in \mathcal{O}_{K}$. Define a slash action $\left.\right|_{k, m}$ of $\Gamma^{J}(K)$ on functions $f: \mathbb{H}_{\mathcal{Q}} \times \mathcal{Q} \rightarrow \mathbb{C}$ by

$$
\begin{aligned}
& \left(\left.f\right|_{k, m}\left[\left(\begin{array}{ll}
\alpha & \beta \\
\gamma & \delta
\end{array}\right),(\lambda, \mu)\right]\right)(\tau, z):=f\left(\left[\left(\begin{array}{cc}
\alpha & \beta \\
\gamma & \delta
\end{array}\right),(\lambda, \mu)\right] \circ(\tau, z)\right) \\
& \quad \times \mathcal{N}(\gamma \tau+\delta)^{-k} \exp \left\{2 \pi i \mathcal{T}_{\mathcal{R}}\left[{ }^{t} m\left(-\widetilde{z^{*}}(\gamma \tau+\delta)^{-1} \gamma z^{*}+\lambda \tau \lambda+2 \lambda z\right) m\right]\right\},
\end{aligned}
$$


where $z^{*}=(z+\tau \lambda+\mu)$. (In fact this action depends only on ${ }^{t} m m$ and ${ }^{t} m \bar{m}$, but it will be convenient to define it in terms of $m$.)

As indicated above, we use the coordinates $(x, y, u, v)$ on $\mathbb{H}_{\mathcal{Q}} \times \mathcal{Q}$ defined by $(\tau, z)=$ $(x+y \mathbf{k}, u+v \mathbf{k})$, where $x, u, v \in \mathbb{C}$ and $y \in \mathbb{R}^{+}$. In Section 5 we will see that the following two differential operators are a basis for the space of second order operators on $\mathbb{H}_{\mathcal{Q}} \times \mathcal{Q}$ invariant with respect to the $(k, m)$-slash action of $\mathrm{SL}_{2}(\mathbb{C}) \ltimes \mathbb{C}^{2}$ :

$$
\begin{aligned}
D_{k, m}^{z}:= & y\left(\partial_{u} \partial_{\bar{u}}+\partial_{v} \partial_{\bar{v}}\right)+8 \pi i \operatorname{Re}\left({ }^{t} \overline{m m} v \partial_{u}\right)-8 \pi^{t} m \bar{m} \operatorname{Re}\left(v \partial_{v}\right) \\
& +16 \pi^{2}\left(\left({ }^{t} m \bar{m}\right)^{2}-\left|{ }^{t} m m\right|^{2}\right) v \bar{v} / y \\
D_{k, m}^{\tau}:= & 4 y^{2} \partial_{x} \partial_{\bar{x}}+y^{2} \partial_{y}^{2}+(2 k-1) y \partial_{y} \\
& +8 y \operatorname{Re}\left(v \partial_{x} \partial_{\bar{u}}\right)+4 y \partial_{y} \operatorname{Re}\left(v \partial_{v}\right) \\
& +4 v \bar{v}\left(\partial_{u} \partial_{\bar{u}}+\partial_{v} \partial_{\bar{v}}\right)-4 \operatorname{Im}^{2}\left(v \partial_{v}\right)+4(k-1) \operatorname{Re}\left(v \partial_{v}\right) \\
& +16 \pi i \operatorname{Re}\left({ }^{t} \overline{m m} v^{2} \partial_{x}\right)-8 \pi^{t} m \bar{m} v \bar{v} \partial_{y} \\
& +16 \pi i(v \bar{v} / y) \operatorname{Re}\left({ }^{t} \overline{m m} v \partial_{u}\right)-16 \pi{ }^{t} m \bar{m}(v \bar{v} / y) \operatorname{Re}\left(v \partial_{v}\right) \\
& +16 \pi^{2}\left(\left({ }^{t} m \bar{m}\right)^{2}-\left|{ }^{t} m m\right|^{2}\right)(v \bar{v} / y)^{2}-4(2 k-1) \pi{ }^{t} m \bar{m} v \bar{v} / y .
\end{aligned}
$$

As in [21] and [19], we will construct functions $f: \mathbb{H}_{\mathcal{Q}} \times \mathcal{Q} \rightarrow \mathbb{C}$ which are $(k, m)$ slash invariant and, in addition, are eigenfunctions of the invariant operator $D_{k, m}^{z}$. (Note that our notation differs slightly from the notation in [21] and [19].) The following definition is analogous to Definition 4.1.7 in Berndt and Schmidt [1]:

DEFINITION 2.1. A $\mathbb{C}^{\infty}$-function $f: \mathbb{H}_{\mathcal{Q}} \times \mathcal{Q} \rightarrow \mathbb{C}$ is a $(k, m)$-Maass-Jacobi form over $K$ if

(i) $\left(\left.f\right|_{k, m} A\right)(\tau, z)=f(\tau, z)$ for all $A \in \Gamma^{J}(K)$.

(ii) There exists $\lambda \in \mathbb{C}$ such that $D_{k, m}^{z} f=\lambda f$.

(iii) $f y^{-k}$ is bounded in domains of type $y \geq y_{0}, y_{0}>0$.

Remark: The theta functions in [21] and [19] are explicit examples of $(k, m)$-MaassJacobi forms (on a subgroup of $\Gamma^{J}(K)$ ) whenever the index vector $m$ is either real or pure imaginary. Such theta functions are, in addition, $(k, m)$-slash invariant eigenfunctions of the invariant operator $D_{k, m}^{\tau}$ if (and only if) $k=1 / 2$. In particular, the theta function $\Theta(\tau, z)$ in Section 3.3 is a simultaneous $(1 / 2, \mathbf{1})$-slash invariant eigenfunction of the two invariant operators $D_{1 / 2, \mathbf{1}}^{z}$ and $D_{1 / 2, \mathbf{1}}^{\tau}$, where 1 denotes a vector such ${ }^{t} \mathbf{1 1}=1$. Note that by Lemma $5.5, y^{-k+1 / 2} \Theta(\tau, z)$ is a simultaneous $(k, \mathbf{1})$-slash invariant eigenfunction of the two invariant operators $D_{k, \mathbf{1}}^{z}$ and $D_{k, \mathbf{1}}^{\tau}$. However, it is not clear if there are any further examples of simultaneous $(k, m)$-slash invariant eigenfunctions of the two invariant operators $D_{k, m}^{z}$ and $D_{k, m}^{\tau}$. 


\section{Eisenstein series}

We now discuss Jacobi-Eisenstein series, which are explicit examples of $(k, m)$ Maass-Jacobi forms (and which are not eigenfunctions of the invariant operator $D_{k, m}^{\tau}$ ). Throughout this section we assume $k \in \mathbb{Z}, m \in \mathbb{R}^{l}$, and ${ }^{t} m m \in \mathbb{N}$. In this setting the term ${ }^{t} m m$ factors out of the argument of the $\mathcal{T}_{\mathcal{R}}$ in the definition of the $(k, m)$-slash action, and so the theory depends only on the scalar ${ }^{t} m m$. For example, the formula for $D_{k, m}^{z}$ reduces to

$$
D_{k, m}^{z}=y\left(\partial_{u} \partial_{\bar{u}}+\partial_{v} \partial_{\bar{v}}\right)+8 \pi i^{t} m m \operatorname{Re}\left(v \partial_{u}\right)-8 \pi^{t} m m \operatorname{Re}\left(v \partial_{v}\right)
$$

and the formula for $D_{k, m}^{\tau}$ simplifies similarly.

For $s \in \mathbb{C}$, formally define the Jacobi-Eisenstein series

$$
E_{k, m, s}(\tau, z):=\sum_{A \in \Gamma_{\infty}^{J}(K) \backslash \Gamma^{J}(K)}\left(\left.y^{s-k+\frac{1}{2}}\right|_{k, m} A\right)(\tau, z),
$$

where $\Gamma_{\infty}^{J}(K)=\left\{\left[\left(\begin{array}{ll}1 & \eta \\ 0 & 1\end{array}\right),(0, n)\right] \mid \eta, n \in \mathcal{O}_{K}\right\}$. It is easy to see that a set of representatives of $\Gamma_{\infty}^{J}(K) \backslash \Gamma^{J}(K)$ is given by $\left\{\left[\left(\begin{array}{cc}\alpha & \beta \\ \gamma & \delta\end{array}\right),(\lambda \alpha, \lambda \beta)\right]\right\}$, where $(\gamma, \delta)$ runs through all elements of $\mathcal{O}_{K} \times \mathcal{O}_{K}$ with $(\gamma, \delta)=1$, i.e., the ideal generated by $\gamma$ and $\delta$ is equal to $\mathcal{O}_{K}, \alpha$ and $\beta$ are chosen such that $\left(\begin{array}{ll}\alpha & \beta \\ \gamma & \delta\end{array}\right) \in \Gamma$, and $\lambda \in \mathcal{O}_{K}$. A straightforward computation shows that

$$
\begin{array}{r}
E_{k, m, s}(\tau, z) \\
=y^{s-k+\frac{1}{2}} \sum_{\substack{\gamma, \delta \in \mathcal{O}_{K} \\
(\gamma, \delta)=1}} \sum_{\lambda \in \mathcal{O}_{K}} \mathcal{N}(\gamma \tau+\delta)^{-s-\frac{1}{2}} \exp \left\{2 \pi i ^ { t } m m \mathcal { T } _ { \mathcal { R } } \left[-\tilde{z}(\gamma \tau+\delta)^{-1} \gamma z\right.\right. \\
\left.\left.+2 \tilde{z}(\gamma \tau+\delta)^{-1} \lambda+\lambda(\alpha \tau+\beta)(\gamma \tau+\delta)^{-1} \lambda\right]\right\}
\end{array}
$$

where $\alpha$ and $\beta$ are chosen so that $\left(\begin{array}{ll}\alpha & \beta \\ \gamma & \delta\end{array}\right) \in \Gamma$. In Section 3.1 we will show that $E_{k, m, s}$ is absolutely convergent for $\operatorname{Re}(s)>7 / 2$. If in addition, $k$ is even, then the expression in (6) yields that $\lim _{y \rightarrow \infty} E_{k, m, k-1 / 2}(y \mathbf{k}, 0)=1$ (see also Section 2 of Ziegler [25]). In particular, the Jacobi-Eisenstein series $E_{k, m, s}(\tau, z)$ does non vanish identically. In the domain of absolute convergence $E_{k, m, s}(\tau, z)$ is invariant under (2), and it is annihilated by $D_{k, m}^{z}$. In Section 3.2 we will give the Fourier expansion of the Eisenstein series and in Section 3.3 we will demonstrate (in the special case that $K=\mathbb{Q}(i)$ and $\left.{ }^{t} m m=1\right)$ that $E_{k, m, s}(\tau, z)$ has a meromorphic continuation to the entire $s$-plane.

3.1. Convergence. We will regard $E_{k, m, s}$ as a subseries of the Jacobi-Eisenstein series of higher degree defined in Section 2 of [25], and show that it converges absolutely if $\operatorname{Re}(s)>7 / 2$. Let $\left\{\omega_{1}, \omega_{2}\right\}$ be an integral basis of $\mathcal{O}_{K}$ and set $\Omega:=\left(\begin{array}{c}\omega_{1} \\ \omega_{1}\end{array} \frac{\omega_{2}}{\omega_{2}}\right)$. Note that $\Omega^{-1}=\left(\begin{array}{cc}\nu_{1} & \bar{\nu}_{1} \\ \nu_{2} & \nu_{2}\end{array}\right)$, where $\left\{\nu_{1}, \nu_{2}\right\}$ is an integral basis for $\mathfrak{d}_{K}^{-1}$. Furthermore, for $\tau=x+y \mathbf{k} \in \mathbb{H}_{\mathcal{Q}}$, let $Z^{*}:=\left(\begin{array}{cc}x & i y \\ i y & \frac{1}{x}\end{array}\right)$ and $Z:={ }^{t} \Omega Z^{*} \Omega$. It is not difficult to see that $Z \in \mathbb{H}_{2}$, the Siegel upper half space of degree 2 (see [18]). If $\eta \in \mathbb{C}$, then let $\operatorname{diag}(\eta)=\left(\begin{array}{ll}\eta & 0 \\ 0 & \bar{\eta}\end{array}\right)$. For $\left(\begin{array}{cc}\alpha & \beta \\ \gamma & \delta\end{array}\right) \in \Gamma$, set $\left(\begin{array}{ll}A^{*} & B^{*} \\ C^{*} & D^{*}\end{array}\right)=\left(\begin{array}{l}\operatorname{diag}(\alpha) \operatorname{diag}(\beta) \\ \operatorname{diag}(\gamma) \operatorname{diag}(\delta)\end{array}\right)$ and 
$\left(\begin{array}{cc}A & B \\ C & D\end{array}\right)=\left(\begin{array}{cc}{ }^{t} \Omega A^{* t} \Omega^{-1} & { }^{t} \Omega B^{*} \Omega \\ \Omega^{-1} C^{* t} \Omega^{-1} & \Omega^{-1} D^{*} \Omega\end{array}\right)$. As in $[18]$

$$
\tau \mapsto\left(\begin{array}{cc}
\alpha & \beta \\
\gamma & \delta
\end{array}\right) \circ \tau \in \mathbb{H}_{\mathcal{Q}} \quad \text { corresponds to } \quad Z \mapsto(A Z+B)(C Z+D)^{-1} \in \mathbb{H}_{2},
$$

and if $\left(\begin{array}{ll}\alpha & \beta \\ \gamma & \delta\end{array}\right) \in \Gamma_{0}\left(\mathfrak{d}_{K}\right):=\left\{\left(\begin{array}{ll}\alpha & \beta \\ \gamma & \delta\end{array}\right) \in \Gamma \mid \gamma \in \mathfrak{d}_{K}\right\}$, then $\left(\begin{array}{cc}A & B \\ C & D\end{array}\right) \in \operatorname{Sp}_{4}(\mathbb{Z})$, the symplectic group over the integers. Moreover, we find that

$$
\begin{aligned}
\mathcal{N}(\gamma \tau+\delta) & =\operatorname{det}(C Z+D), \\
\mathcal{T}_{\mathcal{R}}\left[\tilde{z}(\gamma \tau+\delta)^{-1} \gamma z\right] & =W(C Z+D)^{-1} C{ }^{t} W, \\
\mathcal{T}_{\mathcal{R}}\left[\tilde{z}(\gamma \tau+\delta)^{-1} \lambda\right] & =W(C Z+D)^{-1}{ }^{t} \Lambda, \\
\mathcal{T}_{\mathcal{R}}\left[\lambda(\alpha \tau+\beta)(\gamma \tau+\delta)^{-1} \lambda\right] & =\Lambda(A Z+B)(C Z+D)^{-1}{ }^{t} \Lambda,
\end{aligned}
$$

where ${ }^{t} W={ }^{t} \Omega\left(\begin{array}{l}\|z\|_{\mathbb{C}} \\ \|z\|_{\mathbb{C}}\end{array}\right) \in \mathbb{C}^{2}$ and ${ }^{t} \Lambda=\Omega^{-1}\left(\frac{\lambda}{\lambda}\right) \in \mathbb{Z}^{2}$.

Note that $\Gamma_{0}\left(\mathfrak{d}_{K}\right)$ has finite index in $\Gamma$ and hence (7), (8), (9), and (10) imply that $E_{k, m, k-1 / 2}$ can be regarded as a finite sum of subseries of the Jacobi-Eisenstein series $E_{k, t_{m m}}^{(2)}(Z, W)$ in [25]. Theorem 2.1 in [25] yields that $E_{k, m, k-1 / 2}$ converges absolutely if $k>4$. Hence $E_{k, m, s}$ converges absolutely if $\operatorname{Re}(s)>7 / 2$.

3.2. Fourier expansion. The following proposition gives the Fourier expansion of the above Eisenstein series.

Proposition 3.1. The function $E_{k, m, s}$ has a Fourier expansion of the form

$$
y^{-s+k-\frac{1}{2}} E_{k, m, s}(\tau, z)=\sum_{n^{\prime}, r^{\prime} \in \mathfrak{o}_{K}^{-1}} a_{n^{\prime}, r^{\prime}, m}(y, v) \exp \left\{2 \pi i \mathcal{T}_{\mathcal{R}}\left[n^{\prime} x+r^{\prime} u\right]\right\} .
$$

The Fourier coefficients $a_{n^{\prime}, r^{\prime}, m}(y, v)$ are given by

$$
a_{n^{\prime}, r^{\prime}, m}(y, v)=\delta_{n^{\prime}, r^{\prime}, m} \cdot\left|\mathcal{O}_{K}^{*}\right|+\frac{1}{d_{K}} \sum_{\gamma \in \mathcal{O}_{K}} \mathcal{N}(\gamma)^{-s-\frac{1}{2}} \cdot H_{m, \gamma}\left(n^{\prime}, r^{\prime}\right) \cdot K\left(m, n^{\prime}, r^{\prime}\right)
$$

Here we define $\delta_{n^{\prime}, r^{\prime}, m}$ to be $\exp \left\{2 \pi i \mathcal{T}_{\mathcal{R}}\left[n^{\prime} v \mathbf{k}+\sqrt{r^{\prime} \bar{r}^{\prime}} y \mathbf{k}\right]\right\}$ if there exists $\lambda \in \mathcal{O}_{K}$ such that $n^{\prime}=2 \lambda^{t} m m, r^{\prime}={ }^{t} m m \lambda^{2}$, and 0 otherwise. We also define

$$
H_{m, \gamma}\left(n^{\prime}, r^{\prime}\right):=\sum_{\substack{\delta \in\left(\mathcal{O}_{K} / \gamma \mathcal{O}_{K}\right)^{*} \\ \lambda \in\left(\mathcal{O}_{K} / \gamma \mathcal{O}_{K}\right)}} \exp \left\{2 \pi i \mathcal{T}_{\mathcal{R}}\left[\gamma^{-1}\left({ }^{t} m m \delta^{-1} \lambda^{2}+\delta n^{\prime}-\lambda r^{\prime}\right)\right]\right\},
$$

where $\delta^{-1}$ denotes the inverse of $\delta$ modulo $\gamma$ and

$$
K\left(m, n^{\prime}, r^{\prime}\right):=\int_{\mathbb{C} \times \mathbb{C}} \mathcal{N}(\tau)^{-s-\frac{1}{2}} \exp \left\{-2 \pi i \mathcal{T}_{\mathcal{R}}\left[n^{\prime} x+r^{\prime} u+{ }^{t} m m \tilde{z} \tau^{-1} z\right]\right\} d x d u .
$$

Proof: We use the expression in (6) and distinguish between the cases $\gamma=0$ and $\gamma \neq 0$. The contribution from $\gamma=0$ is

$$
y^{s-k+\frac{1}{2}} \cdot\left|\mathcal{O}_{K}^{*}\right| \sum_{\lambda \in \mathcal{O}_{K}} \exp \left\{2 \pi i{ }^{t} m m \mathcal{T}_{\mathcal{R}}[2 \tilde{z} \lambda+\lambda \tau \lambda]\right\},
$$


which yields the first summand in (11). To compute the contribution from $\gamma \neq 0$, we use the identity

$$
\begin{aligned}
& \mathcal{I}_{\mathcal{R}}\left[-\tilde{z}(\gamma \tau+\delta)^{-1} \gamma z+2 \tilde{z}(\gamma \tau+\delta)^{-1} \lambda+\lambda(\alpha \tau+\beta)(\gamma \tau+\delta)^{-1} \lambda\right] \\
& =\mathcal{T}_{\mathcal{R}}\left[-\left(\tilde{z}-\lambda \gamma^{-1}\right)(\gamma \tau+\delta)^{-1} \gamma\left(z-\lambda \gamma^{-1}\right)+\alpha \gamma^{-1} \lambda^{2}\right],
\end{aligned}
$$

which can be easily verified. Replacing $\lambda$ by $\lambda+\gamma l$ and $\delta$ by $\delta+\gamma d$, where $l, d \in \mathcal{O}_{K}$, $\lambda \in\left(\mathcal{O}_{K} / \gamma \mathcal{O}_{K}\right)$, and $\delta \in\left(\mathcal{O}_{K} / \gamma \mathcal{O}_{K}\right)^{*}$, respectively, we find that the contribution from $\gamma \neq 0$ is given by

$$
\begin{aligned}
& \sum_{\substack{\gamma \in \mathcal{O}_{K} \\
\gamma \neq 0}} \mathcal{N}(\gamma)^{-s-\frac{1}{2}} \sum_{\substack{\delta \in\left(\mathcal{O}_{K} / \gamma \mathcal{O}_{K}\right)^{*} \\
\lambda \in\left(\mathcal{O}_{K} / \gamma \mathcal{O}_{K}\right)}} \exp \left\{2 \pi i^{t} m m \mathcal{T}_{\mathcal{R}}\left[\alpha \gamma^{-1} \lambda^{2}\right]\right\} \sum_{d, l \in \mathcal{O}_{K}} \mathcal{N}\left(\tau+\delta \gamma^{-1}+d\right)^{-s-\frac{1}{2}} \\
& \quad \times \exp \left\{2 \pi i^{t} m m \mathcal{T}_{\mathcal{R}}\left[-\left(\tilde{z}-l-\lambda \gamma^{-1}\right)\left(\tau+\delta \gamma^{-1}+d\right)^{-1}\left(z-l-\lambda \gamma^{-1}\right)\right]\right\} .
\end{aligned}
$$

To complete the proof, it will suffice to compute the Fourier expansion of

$$
\mathcal{F}(\tau, z):=\sum_{d, l \in \mathcal{O}_{K}} \mathcal{N}(\tau+d)^{-s-\frac{1}{2}} \exp \left\{2 \pi i{ }^{t} m m \mathcal{T}_{\mathcal{R}}\left[-(\tilde{z}-l)(\tau+d)^{-1}(z-l)\right]\right\} .
$$

This function is periodic with respect to $\mathcal{O}_{K} \times \mathcal{O}_{K}$, so it has a Fourier expansion

$$
\begin{gathered}
\mathcal{F}(\tau, z)=\sum_{n^{\prime}, r^{\prime} \in \mathfrak{d}_{K}^{-1}} a_{n^{\prime}, r^{\prime}}(y, v) \exp \left\{2 \pi i \mathcal{T}_{\mathcal{R}}\left[n^{\prime} x+r^{\prime} u\right]\right\}, \quad \text { where } \\
a_{n^{\prime}, r^{\prime}}(y, v)=\frac{1}{d_{K}} \int_{\mathbb{C} \times \mathbb{C}} \mathcal{N}(\tau)^{-s-\frac{1}{2}} \exp \left\{-2 \pi i \mathcal{T}_{\mathcal{R}}\left[n^{\prime} x+r^{\prime} u+{ }^{t} m m \tilde{z} \tau^{-1} z\right]\right\} d x d u .
\end{gathered}
$$

3.3. Meromorphic continuation. According to general theory, Eisenstein series have a meromorphic continuation and satisfy a certain matrix functional equation. However, one cannot apply the Langlands theory directly, since the Jacobi group of $K$ is not reductive. In the special case that $K=\mathbb{Q}(i)$ and ${ }^{t} m m=1$, we will sketch a very elementary proof that $E_{k, m, s}$ has a meromorphic continuation to the entire $s$-plane. Bernhard Heim kindly informed the authors that he has used a similar idea in a different setting; for more details see [8]. For the remainder of this section let $K=\mathbb{Q}(i)$ and let $\mathbf{1}$ denote a vector such ${ }^{t} \mathbf{1 1}=1$. Consider the Jacobi theta function

$$
\Theta(\tau, z):=\sum_{\lambda \in \mathcal{O}_{K}} \exp \left\{2 \pi i \mathcal{T}_{\mathcal{R}}[\lambda \tau \lambda+2 \lambda z]\right\}
$$

If $[M,(\lambda, \mu)] \in \Gamma_{0}\left(4 \mathfrak{d}_{K}\right) \ltimes \mathcal{O}_{K}^{2}$, then (see Theorem 3 in [19])

$$
\left(\left.\Theta\right|_{\frac{1}{2}, \mathbf{1}}[M,(\lambda, \mu)]\right)(\tau, z)=\chi(M) \Theta(\tau, z),
$$

where $\chi(M)$ is an eighth root of unity. In Section 4 of [18], $\chi(M)$ is determined

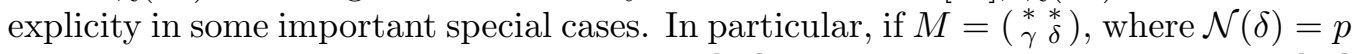
is an odd rational prime, then the results in [18] together with Proposition 4.6 of [12] imply that

$$
\chi\left(\left(\begin{array}{cc}
* & * \\
\gamma & \delta
\end{array}\right)\right)=\left(\frac{\gamma}{\delta}\right)
$$


where $(\dot{\bar{\delta}})$ is the quadratic symbol over $\mathcal{O}_{K}$. Note that (13) holds for all quadratic fields, but the formula for the root of unity in (14) is more complicated for an arbitrary complex quadratic field. Furthermore, if $\eta \in \mathcal{O}_{K}$, then $\Theta(\tau+\eta, z)=\Theta(\tau, z)$

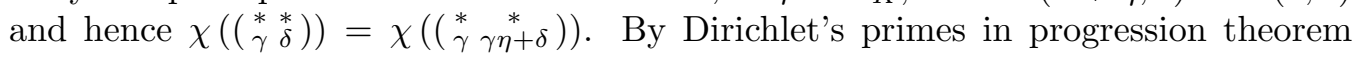
for number fields (see Hecke [7]) the arithmetic progression $\{\gamma \eta+\delta\}_{\eta \in \mathcal{O}_{K}}$ contains infinitely many first degree primes, which yields that (14) holds for all $M \in \Gamma_{0}\left(4 \mathfrak{d}_{K}\right)$, i.e, for $\left[\left(\begin{array}{cc}\alpha & \beta \\ \gamma & \delta\end{array}\right),(\lambda, \mu)\right] \in \Gamma_{0}\left(4 \mathfrak{d}_{K}\right) \ltimes \mathcal{O}_{K}^{2}$ we have

$$
\left(\left.\Theta\right|_{\frac{1}{2}, \mathbf{1}}\left[\left(\begin{array}{cc}
\alpha & \beta \\
\gamma & \delta
\end{array}\right),(\lambda, \mu)\right]\right)(\tau, z)=\left(\frac{\gamma}{\delta}\right) \Theta(\tau, z) .
$$

It is easy to check that (6) can be rewritten as

$$
E_{k, \mathbf{1}, s}(\tau, z)=y^{s-k+\frac{1}{2}} \sum_{\substack{\gamma, \delta \in \mathcal{O}_{K} \\(\gamma, \delta)=1}} \mathcal{N}(\gamma \tau+\delta)^{-s}\left(\left.\Theta\right|_{\frac{1}{2}, \mathbf{1}}[M,(0,0)]\right)(\tau, z) .
$$

Let $M_{j}=\left(\begin{array}{cc}* & * \\ \gamma_{j} & \delta_{j}\end{array}\right)$ be a (finite) set of representatives of right cosets of $\Gamma$ modulo $\Gamma_{0}\left(4 \mathfrak{d}_{K}\right)$, i.e., $\Gamma=\cup \Gamma_{0}\left(4 \mathfrak{d}_{K}\right) M_{j}$. We apply (15) in (16) and write $\left(\tau_{j}, z_{j}\right):=$ $\left[\left(M_{j},(0,0)\right] \circ(\tau, z)\right.$ to find that

$$
E_{k, \mathbf{1}, s}(\tau, z)=y^{s-k+\frac{1}{2}} \sum_{j} \mathcal{N}\left(\gamma_{j} \tau+\delta_{j}\right)^{-s}\left(\left.\Theta\right|_{\frac{1}{2}, \mathbf{1}}\left[M_{j},(0,0)\right]\right)(\tau, z) E\left(s, \tau_{j}\right),
$$

where

$$
E(s, \tau):=\sum_{\substack{\gamma \in 4 \mathfrak{d}_{K} \\ \delta \in \mathcal{O}_{K} \\(\gamma, \delta)=1}}\left(\frac{\gamma}{\delta}\right) \mathcal{N}(\gamma \tau+\delta)^{-s} .
$$

Now one can proceed exactly as in Shimura [20] to show that the Eisenstein series in (18) has a meromorphic continuation to the entire $s$-plane. This is accomplished by determining the Fourier expansion of $E(s, \tau)$. The integrals that occur are well known (see for example [4]) and lead to $K$-Bessel functions which have a meromorphic continuation and which are of exponential decay. In addition, the proof of the meromorphic continuation of $E(s, \tau)$ relies on exact formulas of Gauss sums over $\mathbb{Q}(i)$ and on the continuation of certain $L$-functions. We omit the details.

The sum in (17) is finite and we conclude that the Eisenstein series $E_{k, \mathbf{1}, s}(\tau, z)$ has a meromorphic continuation to the entire $s$-plane.

\section{Invariant differential operators}

The remainder of the paper is devoted to the representation theory and invariant theory necessary to deduce the differential operators $D_{k, m}^{z}$ and $D_{k, m}^{\tau}$ given in (3) and (4). In this section we establish the general framework in which invariant differential operators are computed. This material is known to representation theorists, but to our knowledge there is no one reference in which one can find all of it together.

Fix a real Lie group $G$, a closed subgroup $K$, and a representation $\sigma$ of $K$ on a complex finite dimensional vector space $V$. We will usually write simply $k v$ for $\sigma(k) v$. The complex $G$-vector bundle $G \times_{K} V$ over the homogeneous space $G / K$ is defined to be the set of equivalence classes in $G \times V$ of the relation $(g k, v) \sim(g, k v)$ for all $k \in K$. We will write $[g, v]$ for the equivalence class of $(g, v)$. The projection to $G / K$ 
is of course $[g, v] \mapsto g K$, and the $G$-structure is given by left multiplication in the first coordinate: $g^{\prime}[g, v]=\left[g^{\prime} g, v\right]$.

Write $C^{\infty}\left(G / K: G \times_{K} V\right)$ for the space of smooth sections of $G \times_{K} V$. Suppose that $W$ is another complex finite dimensional representation of $K$. The smooth $W$ valued differential operators on $G \times{ }_{K} V$, i.e., the differential operators from $C^{\infty}(G / K$ : $\left.G \times_{K} V\right)$ to $C^{\infty}\left(G / K: G \times_{K} W\right)$, may themselves be regarded as sections of a $G$ vector bundle over $G / K$. In order to define this bundle, let us use the standard notation $\mathfrak{g}_{0}$ for the real Lie algebra of $G$ and $\mathfrak{g}$ for its complexification:

$$
\mathfrak{g}_{0}:=\operatorname{Lie}_{\mathbb{R}}(G), \quad \mathfrak{g}:=\mathfrak{g}_{0} \otimes \mathbb{C} .
$$

Write $\mathfrak{U}(\mathfrak{g})$ for the universal enveloping algebra of $\mathfrak{g}$ and $\mathfrak{U}^{r}(\mathfrak{g})$ for its degree filtration. As usual, define

$$
\mathfrak{U}(\mathfrak{g}) \otimes_{\mathfrak{k}} V:=(\mathfrak{U}(\mathfrak{g}) \otimes V) / \operatorname{Span}_{\mathbb{C}}\{Z Y \otimes v-Z \otimes Y v: Z \in \mathfrak{U}(\mathfrak{g}), Y \in \mathfrak{k}, v \in V\} .
$$

This space is a $\mathfrak{g}$-module under left multiplication, which does not preserve the finite dimensional filtration $\mathfrak{U}^{r}(\mathfrak{g}) \otimes_{\mathfrak{k}} V$. However, the restriction of this action to $\mathfrak{k}$ is equal to the ad $\otimes \sigma$ action, which does preserve the filtration and so lifts to a $K$-action.

Proposition 4.1. For any two complex finite dimensional representations $V$ and $W$ of $K$, there is a $G$-covariant linear isomorphism from the space of sections

$$
C^{\infty}\left(G / K: G \times_{K}\left[W \otimes\left(\mathfrak{U}(\mathfrak{g}) \otimes_{\mathfrak{k}} V^{*}\right)\right]\right)
$$

to the space of smooth $W$-valued differential operators on $G \times_{K} V$. It carries the degree filtration of $\mathfrak{U}$ to the order filtration of the differential operators, and it respects composition up to symbol.

Proof: The space of sections $C^{\infty}\left(G / K: G \times_{K} V\right)$ may be regarded as the space $C^{\infty}(G: V)^{K}$ of smooth functions $f: G \rightarrow V$ such that $f\left(g k^{-1}\right)=k f(g)$ for all $g \in G$ and $k \in K$. Given an element $\left[g, w \otimes\left(Z \otimes_{\mathfrak{k}} \lambda\right)\right]$ of $G \times_{K}\left(W \otimes\left(\mathfrak{U}(\mathfrak{g}) \otimes_{\mathfrak{k}} V^{*}\right)\right)$, we define a differential operator $D\left[g, w \otimes\left(Z \otimes_{\mathfrak{k}} \lambda\right)\right]$ supported at $g K$ from $C^{\infty}\left(G / K: G \times_{K} V\right)$ to $W$ by

$$
D\left[g, w \otimes\left(Z \otimes_{\mathfrak{k}} \lambda\right)\right] f:=w \lambda(f(g Z)),
$$

where $f(g Z)$ denotes $(\rho(Z) f)(g), \rho$ being the right regular action. It is easy to check that $D$ defines a linear isomorphism with the required properties.

Let us write $\mathbb{D}(G / K, V, W)$ for the space of smooth $G$-covariant differential operators from smooth sections of $G \times{ }_{K} V$ to smooth sections of $G \times_{K} W$. For any group $H$ and any representation $M$ of $H$, we will always write $M^{H}$ for the $H$-fixed vectors of $M$. The following corollary of Proposition 4.1 is immediate from the well-known fact that $C^{\infty}\left(G / K: G \times_{K} V\right)^{G}$ is in canonical bijection with $V^{K}$.

COROllary 4.2. There is a linear isomorphism from $\left(W \otimes\left(\mathfrak{U}(\mathfrak{g}) \otimes_{\mathfrak{k}} V^{*}\right)\right)^{K}$ to $\mathbb{D}(G / K, V, W)$. It carries the degree filtration of $\mathfrak{U}$ to the order filtration of $\mathbb{D}$ and respects composition up to symbol. 
4.1. The $\mathfrak{k}$-split case. Henceforth we restrict to the setting of [11]: we assume that there exists a $K$-splitting $\mathfrak{k} \oplus \mathfrak{m}$ of $\mathfrak{g}$. In this case the Poincaré-Birkhoff-Witt theorem shows that $\mathfrak{U}(\mathfrak{g})$ is $K$-equivalent to $\mathcal{S}(\mathfrak{m}) \otimes \mathfrak{U}(\mathfrak{k})$, where $\mathcal{S}(\mathfrak{m})$ denotes the symmetric algebra of $\mathfrak{m}$. It follows that $\mathfrak{U}(\mathfrak{g}) \otimes_{\mathfrak{k}} V$ is $K$-equivalent to $\mathcal{S}(\mathfrak{m}) \otimes V$ for any representation $V$ of $K$, so Corollary 4.2 reduces to:

Corollary 4.3. Suppose that $\mathfrak{g}=\mathfrak{k} \oplus \mathfrak{m}$ is a K-splitting. Then there is a linear isomorphism from $\left(\mathcal{S}(\mathfrak{m}) \otimes W \otimes V^{*}\right)^{K}$ to $\mathbb{D}(G / K, V, W)$ which carries the degree filtration of $\mathcal{S}$ to the order filtration of $\mathbb{D}$ and respects composition up to symbol.

4.2. Line bundles. Let $\chi$ be a 1-dimensional character of $K$, i.e., a smooth homomorphism from $K$ to $\mathbb{C}^{\times}$. Write $\mathbb{C}_{\chi}$ for the associated 1-dimensional representation of $K$ on $\mathbb{C}$. In this paper we will be interested in invariant differential operators from $C^{\infty}\left(G / K: G \times_{K} \mathbb{C}_{\chi}\right)$ to itself. Here Corollary 4.3 reduces to:

COROLlary 4.4. Suppose that $\mathfrak{g}=\mathfrak{k} \oplus \mathfrak{m}$ is a K-splitting and $\chi$ is a 1-dimensional character of $K$. Then there is a linear isomorphism from $\mathcal{S}(\mathfrak{m})^{K}$ to $\mathbb{D}\left(G / K, \mathbb{C}_{\chi}, \mathbb{C}_{\chi}\right)$ which carries the degree filtration of $\mathcal{S}$ to the order filtration of $\mathbb{D}$ and is an algebra isomorphism up to symbol.

4.3. The topologically trivial case. We will be concerned with topologically trivial $G$-line bundles over $G / K$. The space of sections of such a bundle may be identified with $C^{\infty}(G / K)$, and under any such identification the natural action of $G$ on the sections goes over to an action on functions given by a cocycle (see Lemma 4.6 below). We will need the following elementary definitions and lemmas from group cohomology; their proofs are easy exercises and are omitted.

DEFINITION 4.1. A smooth function $\alpha: G \times G / K \rightarrow \mathbb{C}^{\times}$is a scalar 1-cocycle of $G$ on $G / K$ if

$$
\alpha\left(g g^{\prime}, x\right)=\alpha\left(g, g^{\prime} x\right) \alpha\left(g^{\prime}, x\right)
$$

for all $g, g^{\prime} \in G$ and $x \in G / K$. We write $Z^{1}(G, K)$ for the set of all such cocycles. Note that it forms a group under multiplication.

Given any smooth function $b: G / K \rightarrow \mathbb{C}^{\times}$, its coboundary is the function $\partial b$ : $G \times G / K \rightarrow \mathbb{C}^{\times}$defined by

$$
\partial b(g, x):=b(x) / b(g x) .
$$

We write $B^{1}(G, K)$ for the set of all such coboundaries. Note that it is a subgroup of $Z^{1}(G, K)$.

The quotient group $Z^{1} / B^{1}$ is called $H^{1}(G, K)$, the scalar 1-cohomology group of $G$ on $G / K$. Given $\alpha \in Z^{1}$, we write $[\alpha]$ for its cohomology class, i.e., the coset $\alpha B^{1}$. Two cocycles $\alpha$ and $\beta$ are said to be cohomologous if $[\alpha]=[\beta]$.

Lemma 4.5. Associated to any $\alpha \in Z^{1}(G, K)$ is a representation $\lambda_{\alpha}$ of $G$ on $C^{\infty}(G / K)$, defined by

$$
\left(\lambda_{\alpha}(g) f\right)(x):=\alpha\left(g^{-1}, x\right) f\left(g^{-1} x\right) .
$$

The equivalence classes of these representations are in bijection with $H^{1}$ : given two 1-cocycles $\alpha$ and $\beta$, the representations $\lambda_{\alpha}$ and $\lambda_{\beta}$ are equivalent if and only if $[\alpha]=[\beta]$. More precisely, suppose that $[\alpha]=[\beta]$. Then the quotient $\beta / \alpha$ is a 
coboundary $\partial b$ for some smooth function $b: G / K \rightarrow \mathbb{C}^{\times}$, and the endomorphism of $C^{\infty}(G / K)$ defined by multiplication by $b$ is an intertwining map from $\lambda_{\alpha}$ to $\lambda_{\beta}$.

Lemma 4.6. Associated to any $\alpha \in Z^{1}(G, K)$ is a character $\chi_{\alpha}$ of $K$, defined by $\chi_{\alpha}(k):=\alpha(k, e K)$. Two 1-cocycles $\alpha$ and $\beta$ are cohomologous if and only if $\chi_{\alpha}=\chi_{\beta}$. More precisely, if $[\alpha]=[\beta]$ then $\beta / \alpha=\partial b$, where $b$ is defined by $b(g K)=$ $\alpha(g, K) / \beta(g, K)$ (the right hand side depends only on the coset $g K)$.

For each 1-cocycle $\alpha$, the bundle $G \times_{K} \mathbb{C}_{\chi_{\alpha}}$ is topologically trivial and the natural representation of $G$ on the space of sections $C^{\infty}\left(G / K: G \times_{K} \mathbb{C}_{\chi_{\alpha}}\right)$ is equivalent to the representation $\lambda_{\alpha}$ on $C^{\infty}(G / K)$.

We remark that when $G / K$ is simply connected, the map $[\alpha] \mapsto \chi_{\alpha}$ from $H^{1}(G, K)$ to characters is a bijection: given any character $\chi$ of $K$ there exists a unique cohomology class $[\alpha]$ such that $\chi_{\alpha}=\chi$. To construct a representative $\alpha$ of this class, let $A: G \rightarrow \mathbb{C}^{\times}$be any extension of $\chi$ such that $A(g k)=\chi(k) A(g)$ for all $g \in G$ and $k \in K$, and set $\alpha(g, h K):=A(h) / A(g h)$.

4.4. $\lambda_{\alpha}$-invariant operators. Fix a scalar 1 -cocycle $\alpha$ in $Z^{1}(G, K)$. The $\alpha$-slash action $\left.\right|_{\alpha}$ of $G$ on $C^{\infty}(G / K)$ is defined to be the right action associated to $\lambda_{\alpha}$. For $f \in C^{\infty}(G / K), g \in G$, and $x \in G / K$, it is written

$$
\left.f\right|_{\alpha}[g](x):=\left(\lambda_{\alpha}\left(g^{-1}\right) f\right)(x)=\alpha(g, x) f(g x) .
$$

A differential operator $D: C^{\infty}(G / K) \rightarrow C^{\infty}(G / K)$ is said to be $\lambda_{\alpha}$-invariant, or $\alpha$-slash invariant, if $D \circ \lambda_{\alpha}(g)=\lambda_{\alpha}(g) \circ D$ for all $g \in G$. We denote the algebra of all such operators by $\mathbb{D}_{\alpha}(G / K)$. In light of Lemma 4.6 , here Corollary 4.4 becomes:

Corollary 4.7. Suppose that $\mathfrak{g}=\mathfrak{k} \oplus \mathfrak{m}$ is a K-splitting and $\alpha$ is an element of $Z^{1}(G, K)$. Then there exists a linear isomorphism

$$
\mathrm{IDO}_{\alpha}: \mathcal{S}(\mathfrak{m})^{K} \rightarrow \mathbb{D}_{\alpha}(G / K)
$$

which carries the degree filtration of $\mathcal{S}$ to the order filtration of $\mathbb{D}_{\alpha} . \mathrm{IDO}_{\alpha}$ is an algebra isomorphism at the symbol level: given $\Omega_{1}$ and $\Omega_{2}$ in $\mathcal{S}(\mathfrak{m})^{K}$,

$$
\operatorname{Symbol}\left(\operatorname{IDO}_{\alpha}\left(\Omega_{1} \Omega_{2}\right)\right)=\operatorname{Symbol}\left(\operatorname{IDO}_{\alpha}\left(\Omega_{1}\right)\right) \operatorname{Symbol}\left(\operatorname{IDO}_{\alpha}\left(\Omega_{2}\right)\right) \text {. }
$$

The isomorphism $\mathrm{IDO}_{\alpha}$ is not unique; Proposition 4.8 gives the particular choice of $\mathrm{IDO}_{\alpha}$ that we shall use. In the case that $\alpha$ is trivial, our proposition is equivalent to Theorem 2.8 of Helgason [11].

We will need the symmetrizer map Sym : $\mathcal{S}(\mathfrak{g}) \rightarrow \mathfrak{U}(\mathfrak{g})$, which is defined by

$$
\operatorname{Sym}\left(X_{1} \cdots X_{k}\right):=\frac{1}{k !} \sum_{\sigma \in S_{k}} X_{\sigma(1)} \cdots X_{\sigma(k)} .
$$

It is $\mathfrak{g}$-covariant, so its restriction to $\mathcal{S}(\mathfrak{m})$ is an injective $K$-covariant map.

Proposition 4.8. One choice of the isomorphism $\operatorname{IDO}_{\alpha}: \mathcal{S}(\mathfrak{m})^{K} \rightarrow \mathbb{D}_{\alpha}(G / K)$ in Corollary 4.7 is the map defined by

$$
\left(\operatorname{IDO}_{\alpha}(\Omega) f\right)(g K):=\left(\lambda_{\alpha}(g) \lambda_{\alpha}(\operatorname{Sym}(\Omega)) \lambda_{\alpha}\left(g^{-1}\right) f\right)(g K) .
$$


Proof: First one must check that $\mathrm{IDO}_{\alpha}$ is well-defined, i.e., that the right hand side of (20) is independent of the choice of representative of the coset $g K$. Let us write simply $\lambda_{\alpha}\left(g \Omega g^{-1}\right)$ as an abbreviation for $\lambda_{\alpha}(g) \lambda_{\alpha}(\operatorname{Sym}(\Omega)) \lambda_{\alpha}\left(g^{-1}\right)$. If $g$ is replaced by $g k$ for some $k \in K$, then $\lambda_{\alpha}\left(g \Omega g^{-1}\right)$ is replaced by $\lambda_{\alpha}\left(g k \Omega k^{-1} g^{-1}\right)=$ $\lambda_{\alpha}\left(g \operatorname{Ad}_{k}(\Omega) g^{-1}\right)$. Since $\Omega$ is $K$-invariant, there is no change.

To verify that $\operatorname{IDO}_{\alpha}(\Omega)$ is $G$-invariant, note that $\left(\lambda_{\alpha}(h) \operatorname{IDO}_{\alpha}(\Omega) f\right)(g K)$ may be rewritten as

$$
\begin{aligned}
& \alpha\left(h^{-1}, g K\right)\left(\operatorname{IDO}_{\alpha}(\Omega) f\right)\left(h^{-1} g K\right) \\
& \quad=\alpha\left(h^{-1}, g K\right)\left(\lambda_{\alpha}\left(h^{-1} g \Omega g^{-1} h\right) f\right)\left(h^{-1} g K\right) \\
& \quad=\alpha\left(h^{-1}, g K\right) \alpha\left(h, h^{-1} g K\right)\left(\lambda_{\alpha}\left(g \Omega g^{-1} h\right) f\right)(g K) .
\end{aligned}
$$

Applying the cocycle property, one sees that this is $\left(\operatorname{IDO}_{\alpha}(\Omega) \lambda_{\alpha}(h) f\right)(g K)$.

We leave the reader to check that $\operatorname{IDO}_{\alpha}(\Omega)$ is indeed a differential operator of order equal to the degree of $\Omega$, and that $\mathrm{IDO}_{\alpha}$ defines an algebra isomorphism of symbols.

In practice we will use the following somewhat more explicit form of (20):

$$
\left(\operatorname{IDO}_{\alpha}(\Omega) f\right)(g K)=\left.\alpha^{-1}(g, K) \lambda_{\alpha}(\operatorname{Sym}(\Omega))\right|_{x=K}(\alpha(g, x) f(g x)) .
$$

Here the subscript $x=K$ on the right signifies that the operator $\lambda_{\alpha}(\operatorname{Sym}(\Omega))$ differentiates with respect the $\operatorname{coset} x$ and then evaluates at the identity coset $K$, regarding $g$ as a constant.

\section{The complex Jacobi group}

In this section we derive the formulas for the invariant differential operators $D_{k, m}^{\tau}$ and $D_{k, m}^{z}$ given in Section 2, and prove that they span the space of all second order differential operators invariant with respect to the $(k, m)$-slash action. We also prove that the algebra all $(k, m)$-slash invariant differential operators is generated by these two second order and two third order operators, but we will not give explicit formulas for the third order generators.

We begin with the definition of the complex Jacobi group $G$ and a short conceptual proof that (2) is an action. We give two presentations of $G$, the complexifications of the two presentations of the real Jacobi group given in Theorem 1.4 of [3] and Section 1.1 of [1], respectively.

The complex Heisenberg group $\mathbb{C}^{2} \tilde{x} \mathbb{C}$ is the central extension of $\mathbb{C}^{2}$ by $\mathbb{C}$ defined as follows: writing elements of $\mathbb{C}^{2}$ as row vectors $X$ and elements of $\mathbb{C}$ as scalars $\kappa$,

$$
(X, \kappa)\left(X^{\prime}, \kappa^{\prime}\right):=\left(X+X^{\prime}, \operatorname{det}\left(\begin{array}{l}
X \\
X^{\prime}
\end{array}\right)+\kappa+\kappa^{\prime}\right),
$$

where $\operatorname{det}\left(\begin{array}{l}X \\ X^{\prime}\end{array}\right)$ denotes the determinant of the matrix $\left(\begin{array}{l}X \\ X^{\prime}\end{array}\right)$.

The complex Jacobi group $G$ is the semidirect product $\mathrm{SL}_{2} \mathbb{C} \ltimes\left(\mathbb{C}^{2} \tilde{x} \mathbb{C}\right)$ associated to the right action $(X, \kappa) M:=(X M, \kappa)$ of $\mathrm{SL}_{2} \mathbb{C}$ on $\mathbb{C}^{2} \tilde{\times} \mathbb{C}$. Thus its group law is

$$
(M, X, \kappa)\left(M^{\prime}, X^{\prime}, \kappa^{\prime}\right):=\left(M M^{\prime}, X M^{\prime}+X^{\prime}, \operatorname{det}\left(\begin{array}{c}
X M^{\prime} \\
X^{\prime}
\end{array}\right)+\kappa+\kappa^{\prime}\right) .
$$

Note that the center of $G$ is

$$
Z(G):=\{(I, 0, \kappa): \kappa \in \mathbb{C}\} .
$$


In order to give the second presentation of $G$, recall that the complex symplectic group $\mathrm{Sp}_{2 n} \mathbb{C}$ is the group of invertible complex $2 n \times 2 n$ matrices $g$ whose $n \times n$ blocks are $A, B, C$, and $D$ and whose inverse is given by the natural extension of the formula for the inverse of an element of $\mathrm{SL}_{2} \mathbb{C}$ :

$$
g=\left(\begin{array}{cc}
A & B \\
C & D
\end{array}\right), \quad g^{-1}=\left(\begin{array}{cc}
{ }^{t} D & -{ }^{t} B \\
-{ }^{t} C & { }^{t} A
\end{array}\right) .
$$

One checks that there is an injective homomorphism $\pi_{4}: G \rightarrow \mathrm{Sp}_{4} \mathbb{C}$ defined by

$$
\pi_{4}(M, X, \kappa):=\left(\begin{array}{cccc}
M_{11} & 0 & M_{12} & X_{2} \\
X_{1} & 1 & X_{2} & \kappa \\
M_{21} & 0 & M_{22} & -X_{1} \\
0 & 0 & 0 & 1
\end{array}\right)
$$

(this is easier to see after conjugating $\mathrm{Sp}_{4} \mathbb{C}$ by the permutation matrix which exchanges the second and third coordinates). The image $\pi_{4}(G)$ realizes $G$ as the subgroup of $\mathrm{Sp}_{4} \mathbb{C}$ consisting of all those elements whose bottom row is $(0,0,0,1)$.

5.1. The action of $G$ on $\mathbb{H}_{\mathcal{Q}} \times \mathcal{Q}$. Recall the action (1) of $\Gamma^{J}(K)$ on $\mathbb{H}_{\mathcal{Q}} \times \mathcal{Q}$, where $\mathcal{Q}$ is the quaternion skew field and $\mathbb{H}_{\mathcal{Q}}$ is the upper half space of quaternions with positive real $\mathbf{k}$ part. In fact (1) defines a group action of $G / Z(G)=\mathrm{SL}_{2} \mathbb{C} \ltimes \mathbb{C}^{2}$ and hence also of $G$ on $\mathbb{H}_{\mathcal{Q}} \times \mathcal{Q}$. Verifying this directly is somewhat tedious, so we now give a conceptual proof which explains the origin of the action.

Clearly there is a homomorphism $\pi_{3}: G \rightarrow \mathrm{SL}_{3} \mathbb{C}$ with kernel $Z(G)$, defined by

$$
\pi_{3}(M, X, \kappa):=\left(\begin{array}{ccc}
M_{11} & M_{12} & 0 \\
M_{21} & M_{22} & 0 \\
X_{1} & X_{2} & 1
\end{array}\right) .
$$

This homomorphism may be used to define a left action $L_{3}$ of $G$ on the space of row vectors $\mathcal{Q}^{3}$ by

$$
\begin{aligned}
& L_{3}(M, X, \kappa)\left(\tau_{1}, \tau_{2}, \tau_{3}\right):=\left(\tau_{1}, \tau_{2}, \tau_{3}\right){ }^{t} \pi_{3}(M, X, \kappa) \\
& \quad=\left(\tau_{1} M_{11}+\tau_{2} M_{12}, \tau_{1} M_{21}+\tau_{2} M_{22}, \tau_{1} X_{1}+\tau_{2} X_{2}+\tau_{3}\right) .
\end{aligned}
$$

This action commutes with the left scalar action of $\mathcal{Q}$, so it descends to an action on the projective space of quaternionic lines of the form

$$
\left[\tau_{1}, \tau_{2}, \tau_{3}\right]:=\left\{\left(w \tau_{1}, w \tau_{2}, w \tau_{3}\right): w \in \mathcal{Q}\right\} .
$$

One checks that this quotient action preserves the set of lines such that $\tau_{2}^{-1} \tau_{1}$ is in $\mathbb{H}_{\mathcal{Q}}$, which may be identified with $\mathbb{H}_{\mathcal{Q}} \times \mathcal{Q}$ via

$$
\left[\tau_{1}, \tau_{2}, \tau_{3}\right] \mapsto\left(\tau_{2}^{-1} \tau_{1}, \tau_{2}^{-1} \tau_{3}\right) .
$$

Carrying it over to an action of $G$ on $\mathbb{H}_{\mathcal{Q}} \times \mathcal{Q}$ yields $(M, X, \kappa) \circ(\tau, z):=$

$$
\left(\left(\tau M_{21}+M_{22}\right)^{-1}\left(\tau M_{11}+M_{12}\right),\left(\tau M_{21}+M_{22}\right)^{-1}\left(\tau X_{1}+X_{2}+z\right)\right) .
$$

Since $\operatorname{det}(M)$ is real, one finds that

$$
\left(\tau M_{21}+M_{22}\right)^{-1}\left(\tau M_{11}+M_{12}\right)=\left(M_{11} \tau+M_{12}\right)\left(M_{21} \tau+M_{22}\right)^{-1} .
$$

Therefore (1) and (22) define the same action. We have proven most of the following lemma; we leave the rest to the reader. 
Lemma 5.1. The equation (22) defines a transitive action of the group $G$ on $\mathbb{H}_{\mathcal{Q}} \times$ $\mathcal{Q}$. The stabilizer of the element $(\mathbf{k}, 0)$ of $\mathbb{H}_{\mathcal{Q}} \times \mathcal{Q}$ is the subgroup $K:=\mathrm{SU}_{2} \times Z(G)$. Hence $g K \mapsto g \circ(\mathbf{k}, 0)$ defines a diffeomorphism from $G / K$ to $\mathbb{H}_{\mathcal{Q}} \times \mathcal{Q}$.

5.2. The space $\mathcal{S}(\mathfrak{m})^{K}$. By Corollary 4.7, whenever $\mathfrak{k} \subseteq \mathfrak{g}$ is $K$-split the graded algebra of $\mathbb{D}_{\alpha}(G / K)$ is $\mathcal{S}(\mathfrak{m})^{K}$, independent of the cocycle $\alpha$. This turns out to be the case in our setting. Henceforth let us write $\mathcal{S}^{n}(\mathfrak{m})$ for the space of homogeneous elements of $\mathcal{S}(\mathfrak{m})$ of degree $n$. For Section 2 we need only apply the map $\mathrm{IDO}_{\alpha}$ of Proposition 4.8 to $\mathcal{S}^{2}(\mathfrak{m})^{K}$, the quadratic invariants. For the sake of completeness, in this section we will describe the entire algebra $\mathcal{S}(\mathfrak{m})^{K}$.

Since Section 4 applies only to real Lie groups, we must forget that the complex Jacobi group $G$ has a holomorphic structure and regard it as a 12 dimensional real group (indeed, it does not act holomorphically on $\mathbb{H}_{\mathcal{Q}} \times \mathcal{Q}$ ). As such, $\mathfrak{g}_{0}$ is its real Lie algebra and $\mathfrak{g}$ is the complexification of $\mathfrak{g}_{0}$. We find

$$
\mathfrak{g}_{0}=\left\{(M, X, \kappa): M \in \mathfrak{s l}_{2} \mathbb{C}, X \in \mathbb{C}^{2}, \kappa \in \mathbb{C}\right\},
$$

with Lie bracket

$$
\left[(M, X, \kappa),\left(M^{\prime}, X^{\prime}, \kappa^{\prime}\right)\right]=\left(\left[M, M^{\prime}\right], X M^{\prime}-X^{\prime} M, 2 \operatorname{det}\left(\begin{array}{c}
X \\
X^{\prime}
\end{array}\right)\right)
$$

and exponential map

$$
\exp (M, X, \kappa)=\left(e^{M}, X\left(\frac{e^{M}-I}{M}\right), \kappa\right) .
$$

The standard complex basis of $\mathfrak{s l}_{2} \mathbb{C}$ is

$$
H:=\left(\begin{array}{cc}
1 & 0 \\
0 & -1
\end{array}\right), \quad E:=\left(\begin{array}{cc}
0 & 1 \\
0 & 0
\end{array}\right), \quad F:=\left(\begin{array}{ll}
0 & 0 \\
1 & 0
\end{array}\right) .
$$

Define $e, f$, and $Z$ in $\mathfrak{g}_{0}$ by

$$
Z:=(0,(0,0), 1), \quad e:=(0,(0,1), 0), \quad f:=(0,(1,0), 0) .
$$

Regarding $\mathfrak{s l}_{2} \mathbb{C}$ as a subalgebra of $\mathfrak{g}_{0}$, we see that

$$
\{H, i H, E, i E, F, i F, Z, i Z, e, i e, f, i f\}
$$

is a basis of the complexification $\mathfrak{g}$. Since $i$ denotes $\sqrt{-1}$ in the complex structure of $\mathfrak{g}_{0}$, which we are required to forget, we will write $j$ for $\sqrt{-1}$ in the complex structure of $\mathfrak{g}$. To emphasize this we write

$$
\mathbb{C}_{i}:=\{a+b i: a, b \in \mathbb{R}\}, \quad \mathbb{C}_{j}:=\{a+b j: a, b \in \mathbb{R}\} .
$$

The complexified Lie algebra $\mathfrak{k}$ of the stabilizer $K$ of $(\mathbf{k}, 0)$ has a $K$-invariant complement $\mathfrak{m}$ (which is not a Lie algebra). These two spaces are

$$
\begin{aligned}
\mathfrak{k} & =\operatorname{Span}_{\mathbb{C}_{j}}\{i H, F-E, i(F+E), Z, i Z\}, \\
\mathfrak{m} & :=\operatorname{Span}_{\mathbb{C}_{j}}\{H, i(F-E), F+E, e, i e, f, i f\} .
\end{aligned}
$$

Since the center $Z(K)=Z(G)$ acts trivially on $\mathfrak{m}$, the $K$-invariants of $\mathcal{S}(\mathfrak{m})$ are the same as its $\mathrm{SU}_{2}$-invariants. At this point we state some results from the representation theory of $\mathrm{SU}_{2}$; see any book on Lie theory, for example [6]. Up to equivalence, $\mathrm{SU}_{2}$ has a unique irreducible $n+1$-dimensional complex representation $L_{n}$ for all $n \geq 0$. 
One realization of $L_{n}$ is as $\mathcal{S}^{n}\left(\mathbb{C}^{2}\right)$, where $\mathrm{SU}_{2}$ acts in the standard manner on $\mathbb{C}^{2}$. The following formulas are classical:

$$
\begin{aligned}
& \mathcal{S}^{n}\left(L_{1}\right) \cong L_{n}, \quad \mathcal{S}^{n}\left(L_{2}\right) \cong L_{2 n} \oplus L_{2 n-4} \oplus \cdots \oplus L_{2 \operatorname{Res}_{2}(n)}, \\
& L_{n} \otimes L_{m} \cong L_{n+m} \oplus L_{n+m-2} \oplus \cdots \oplus L_{|n-m|},
\end{aligned}
$$

where $\cong$ denotes equivalence and $\operatorname{Res}_{2}$ denotes the residue modulo 2 .

As a representation of $K$, the space $\mathfrak{m}$ decomposes as a direct sum $\mathfrak{m}_{2} \oplus \mathfrak{m}_{1}^{+} \oplus \mathfrak{m}_{1}^{-}$ of irreducible subrepresentations such that $\mathfrak{m}_{2} \cong L_{2}$ and $\mathfrak{m}_{1}^{ \pm} \cong L_{1}$. They are

$$
\mathfrak{m}_{2}=\operatorname{Span}_{\mathbb{C}_{j}}\{H, i(F-E), F+E\}, \quad \mathfrak{m}_{1}^{ \pm}=\operatorname{Span}_{\mathbb{C}_{j}}\{e \pm j i e, f \pm j i f\} .
$$

The next lemma follows easily from (24), except for the relation, which can be deduced from a (not too difficult) calculation in $\mathcal{S}\left(L_{2} \oplus L_{1} \oplus L_{1}\right)$ using the weight bases of the $L_{n}$ regarded as holomorphic representations of $\mathfrak{s l}_{2} \mathbb{C}$. We omit the proof.

Lemma 5.2. There are unique (up to a scalar) non-zero invariants

$$
\begin{aligned}
& Q_{\tau} \in \mathcal{S}^{2}\left(\mathfrak{m}_{2}\right)^{K}, \quad Q_{z} \in\left(\mathfrak{m}_{1}^{+} \otimes \mathfrak{m}_{1}^{-}\right)^{K}, \\
& C_{ \pm} \in\left(\mathfrak{m}_{2} \otimes \mathcal{S}^{2}\left(\mathfrak{m}_{1}^{ \pm}\right)\right)^{K}, \quad C_{0} \in\left(\mathfrak{m}_{2} \otimes \mathfrak{m}_{1}^{+} \otimes \mathfrak{m}_{1}^{-}\right)^{K}
\end{aligned}
$$

These five invariants are a basis of $\mathcal{S}^{2}(\mathfrak{m})^{K} \oplus \mathcal{S}^{3}(\mathfrak{m})^{K}$.

We may (and do) normalize the quadratic invariants so that

$$
Q_{\tau}=H^{2}+(i F-i E)^{2}+(F+E)^{2}, \quad Q_{z}=e^{2}+(i e)^{2}+f^{2}+(i f)^{2} .
$$

We may (and do) normalize the cubic invariants so that

$$
C_{0}^{2}-C_{+} C_{-}-Q_{\tau} Q_{z}^{2}=0 .
$$

Of course, $\mathcal{S}(\mathfrak{m})$ is $\mathbb{C}_{j}$-linear but not $\mathbb{C}_{i}$-linear, so for example $(i e)^{2}$ is not $-e^{2}$. It is not hard to compute formulas for the cubic invariants, but they are not illuminating and we will not need them. We remark that $\mathbb{C}_{i}$-conjugation induces a $\mathbb{C}_{j}$-linear involution on $\mathfrak{m}$ which preserves $\mathfrak{m}_{2}$ and exchanges $\mathfrak{m}_{1}^{ \pm}$. The cubic invariants may be chosen so that this involution acts on $\mathcal{S}(\mathfrak{m})^{K}$ so as to fix $Q_{\tau}, Q_{z}$, and $C_{0}$ and exchange $C_{ \pm}$.

We conclude this section with a theorem giving a complete description of $\mathcal{S}(\mathfrak{m})^{K}$. Recall that the Hilbert series of $\mathcal{S}(\mathfrak{m})^{K}$ is defined to be

$$
H(t):=\sum_{n=0}^{\infty} \operatorname{dimension}\left(\mathcal{S}^{n}(\mathfrak{m})^{K}\right) t^{n}
$$

TheOrem 5.3. The algebra $\mathcal{S}(\mathfrak{m})^{K}$ is generated by $Q_{\tau}, Q_{z}, C_{+}, C_{-}$, and $C_{0}$. The ideal of relations between these generators is generated by $C_{0}^{2}-C_{+} C_{-}-Q_{\tau} Q_{z}^{2}$. Thus

$$
\mathcal{S}(\mathfrak{m})^{K} \cong \mathbb{C}\left[q_{\tau}, q_{z}, c_{+}, c_{-}, c_{0}\right] /\left\langle c_{0}^{2}-c_{+} c_{-}-q_{\tau} q_{z}^{2}\right\rangle,
$$

where $q_{\tau}, q_{z}, c_{+}, c_{-}$, and $c_{0}$ are algebraically independent indeterminates.

The Hilbert series of $\mathcal{S}(\mathfrak{m})^{K}$ is

$$
H(t)=\frac{1+t^{3}}{\left(1-t^{2}\right)^{2}\left(1-t^{3}\right)^{2}}=\frac{1-t^{6}}{\left(1-t^{2}\right)^{2}\left(1-t^{3}\right)^{3}} .
$$


PROOF: We give very brief outlines of two proofs. In the first one begins by using the weight bases of the $L_{n}$ regarded as holomorphic representations of $\mathfrak{s l}_{2} \mathbb{C}$ to compute explicit formulas for the five invariants in Lemma 5.2. An elementary (but slightly tricky) direct calculation then shows that these invariants generate the entire algebra of invariants, and a simpler calculation shows that any relation between them must be a multiple of 25. The formula for the Hilbert series is a corollary of these results.

In the second proof, one begins by proving the formula for $H(t)$. This requires a long calculation using (24) and the fact that

$$
\mathcal{S}^{n}\left(\bigoplus_{i=1}^{r} V_{i}\right)=\bigoplus_{n_{1}+\cdots+n_{r}=n} \bigotimes_{i=1}^{r} \mathcal{S}^{n_{i}}\left(V_{i}\right)
$$

for any vector spaces $V_{i}$. Then one proves that the relation ideal is generated by the cubic relation just as in the first proof. It follows by standard methods from invariant theory (see for example [6] or [22]) that $\mathcal{S}(\mathfrak{m})^{K}$ has two quadratic generators, three cubic generators, and one relation of degree six in $\mathfrak{m}$. (In fact, the formula for the Hilbert series alone probably already implies this.) Lemma 5.2 now completes the proof.

5.3. The second order $\left.\right|_{k, m}$-invariant operators. Corollary 4.7 gives the following corollary of Theorem 5.3 (see Proposition 5.8 for a sharper result).

Corollary 5.4. For any cocycle $\alpha \in Z^{1}(G, K)$, the algebra of invariant differential operators $\mathbb{D}_{\alpha}(G / K)$ is generated by the two second order and three third order operators obtained by applying $\mathrm{IDO}_{\alpha}$ to $Q_{\tau}, Q_{z}, C_{+}, C_{-}$, and $C_{0}$.

In this section we define the cocycles $\alpha_{k, m}$ associated to the slash action defined in (2) and compute the operators (3) and (4). Throughout the calculation we will use Lemma 5.1 to regard $G / K$ as $\mathbb{H}_{\mathcal{Q}} \times \mathcal{Q}$.

Recall from Section 2 the following definitions for $z=u+v \mathbf{k} \in \mathcal{Q}$ :

$$
\tilde{z}:=u+\bar{v} \mathbf{k}, \quad \mathcal{T}_{\mathcal{R}}[z]:=2 \operatorname{Re}(u)+2 i \operatorname{Re}(v), \quad \mathcal{N}(z):=\bar{z} z=|u|^{2}+|v|^{2} .
$$

In order to define $\alpha_{k, m}$, let $a: G \times\left(\mathbb{H}_{\mathcal{Q}} \times \mathcal{Q}\right) \rightarrow \mathbb{C}$ be

$$
\begin{aligned}
& a((M, X, \kappa),(\tau, z)):=\kappa+X_{1} X_{2}+2 X_{1} z+X_{1} \tau X_{1} \\
& \quad-\left(\tilde{z}+X_{1} \tau+X_{2}\right)\left(M_{21} \tau+M_{22}\right)^{-1} M_{21}\left(z+\tau X_{1}+X_{2}\right) .
\end{aligned}
$$

For any $k \in \mathbb{C}$ and $m \in \mathbb{C}^{l}$, define $\alpha_{k, m}: G \times\left(\mathbb{H}_{\mathcal{Q}} \times \mathcal{Q}\right) \rightarrow \mathbb{C}^{\times}$by

$\alpha_{k, m}((M, X, \kappa),(\tau, z)):=\mathcal{N}^{-k}\left(M_{21} \tau+M_{22}\right) \exp \left\{2 \pi i \mathcal{T}_{\mathcal{R}}\left[{ }^{t} m a((M, X, \kappa),(\tau, z)) m\right]\right\}$.

Lemma 5.5. The function $\alpha_{k, m}$ is a 1-cocycle. Moreover, $\alpha_{k, 0}$ is the coboundary $\partial y^{-k}$, where $\tau=x+y \mathbf{k}$. Hence $\alpha_{k, m}=\partial y^{-k} \cdot \alpha_{0, m}$, and multiplication by $y^{-k^{\prime}}$ is an intertwining map from $\lambda_{\alpha_{k, m}}$ to $\lambda_{\alpha_{k+k^{\prime}, m}}$ and thus also from $\left.\right|_{\alpha_{k, m}}$ to $\left.\right|_{\alpha_{k+k^{\prime}, m}}$ (see Sections 4.3 and 4.4). In particular, for any $\Omega \in \mathcal{S}(\mathfrak{m})^{K}$ we have

$$
\operatorname{IDO}_{\alpha_{k+k^{\prime}, m}}(\Omega)=y^{-k^{\prime}} \circ \operatorname{IDO}_{\alpha_{k, m}}(\Omega) \circ y^{k^{\prime}} .
$$

Proof: To prove that $\alpha_{0, m}$ is a cocycle we must prove that

$$
{ }^{t} m\left[a\left(g_{1} g_{2},(\tau, z)\right)-a\left(g_{1}, g_{2} \circ(\tau, z)\right)-a\left(g_{2},(\tau, z)\right)\right] m
$$


is annihilated by $\mathcal{T}_{\mathcal{R}}$ for all $g_{1}, g_{2} \in G$ and $(\tau, z) \in \mathbb{H}_{\mathcal{Q}} \times \mathcal{Q}$. Since $\lambda_{\alpha}$ is a representation, it suffices to check this for each of the $g_{i}$ in either $\mathrm{SL}_{2} \mathbb{C}$ or the Heisenberg group $\mathbb{C}^{2} \tilde{x} \mathbb{C}$. Two of these calculations are easy, but the two with $g_{2} \in \mathrm{SL}_{2} \mathbb{C}$ are rather long. It helps to note that $w \mapsto \tilde{w}$ is an antiautomorphism of $\mathcal{Q}$ which is the identity on both $\mathbb{C}$ and $\mathbb{H}_{\mathcal{Q}}$, and that $\mathcal{T}_{\mathcal{R}}\left[{ }^{t} m(w-\tilde{w}) m\right]=0$ for all $w \in \mathcal{Q}$. (It would be interesting to have a more conceptual proof that $\alpha_{0, m}$ is a cocycle, one which explained its origin.) The remainder of the lemma follows from Lemma 4.5.

We remark that from a purely representation-theoretic point of view, the parameter $k$ is uninteresting because it adds only a coboundary; it is included here for its number-theoretic interest. In addition, the only datum in $m$ of representationtheoretic interest is the complex scalar ${ }^{t} \mathrm{~mm}$. To explain, write the function $a$ as $a_{1}+a_{\mathbf{k}} \mathbf{k}$ with $a_{1}$ and $a_{\mathbf{k}}$ complex. Then

$$
\alpha_{0, m}=\exp \left\{2 \pi i \mathcal{T}_{\mathcal{R}}\left[{ }^{t} m m a_{1}\right]\right\} \exp \left\{2 \pi i \mathcal{T}_{\mathcal{R}}\left[{ }^{t} m \bar{m} a_{\mathbf{k}}\right]\right\},
$$

and the second factor can be shown to be the coboundary of $4 \pi^{t} m \bar{m} v \bar{v} / y$.

We come now to the results needed in the number-theoretic part of the paper: Lemma 5.6 links the cocycle $\alpha_{k, m}$ to the slash action $\left.\right|_{k, m}$ of Section 2, and Proposition 5.7 is the required result on the differential operators (3) and (4).

Recall the complex quadratic field $K$ and the group $\Gamma^{J}(K)$ from Section 2. Define $G(K)$ to be the subgroup $\Gamma \ltimes\left(\mathcal{O}_{K}^{2} \tilde{\times} \mathcal{O}_{K}\right)$ of $G$, a central extension of $\Gamma^{J}(K)$. Check that for ${ }^{t} m m \in \mathcal{O}_{K}$, the terms $\kappa+X_{1} X_{2}$ in $a(g,(\tau, z))$ do not contribute to $\alpha_{k, m}$ for $g \in G(K)$. Therefore the slash action $\left.\right|_{\alpha_{k, m}}$ is trivial on the center $\mathcal{O}_{K}$ of $G(K)$ and hence descends to an action of $\Gamma^{J}(K)$. The following lemma is easy to verify:

Lemma 5.6. For ${ }^{t} m m \in \mathcal{O}_{k},\left.\right|_{\alpha_{k, m}}$ and $\left.\right|_{k, m}$ coincide on $\Gamma^{J}(K)$.

Proposition 5.7. The operators $D_{k, m}^{\tau}$ and $D_{k, m}^{z}$ of Section 2 span the space of second order $\left.\right|_{k, m}$-invariant differential operators. They satisfy

$$
D_{k, m}^{\tau}=\operatorname{IDO}_{\alpha_{k, m}}\left(Q_{\tau} / 4\right), \quad D_{k, m}^{z}=\operatorname{IDO}_{\alpha_{k, m}}\left(Q_{z} / 4\right)+4 \pi^{t} m \bar{m} .
$$

Proof: We need only prove the formulas; the spanning statement then follows from Corollary 5.4 and Lemma 5.6. By Lemma 5.5, the $(k, m)$ operators are given by conjugating the $(0, m)$ operators by $y^{-k}$, so it suffices to consider the case $k=0$. We must apply (21) to the elements $Q_{\tau}$ and $Q_{z}$ of $\mathcal{S}^{2}(\mathfrak{m})^{K}$ given in Lemma 5.2. First we apply the symmetrizer map. Since $Q_{\tau}$ and $Q_{z}$ are sums of squares of elements of $\mathfrak{g}$, $\operatorname{Sym}\left(Q_{\tau}\right)$ and $\operatorname{Sym}\left(Q_{z}\right)$ are given by the same formulas, now viewed as elements of $\mathfrak{U}(\mathfrak{g})^{K}$. We will abuse notation and write $Q_{\tau}$ and $Q_{z}$ for them. Let us also write $\lambda$ for $\lambda_{1}$, the untwisted left action of $G$ on $\mathbb{H}_{\mathcal{Q}} \times \mathcal{Q}$, and $\lambda_{m}$ and $\alpha_{m}$ for $\lambda_{\alpha_{0, m}}$ and $\alpha_{0, m}$, respectively.

Next we need $\left.\lambda_{m}\left(Q_{\tau}\right)\right|_{(\mathbf{k}, 0)}$ and $\left.\lambda_{m}\left(Q_{z}\right)\right|_{(\mathbf{k}, 0)}$. Given any cocycle $\alpha$ and any $X \in \mathfrak{g}$, let $\alpha(X)$ denote the function on $\mathbb{H}_{\mathcal{Q}} \times \mathcal{Q}$ defined by $\left.(\tau, z) \mapsto D\right|_{t=0} \alpha\left(e^{t X},(\tau, z)\right)$. The definitions of $\lambda_{\alpha}$ (see Lemma 4.5) and $\alpha_{m}$ give

$$
\lambda_{\alpha}(X)=\lambda(X)-\alpha(X), \quad \alpha_{m}(X)=2 \pi i \mathcal{T}_{\mathcal{R}}\left[{ }^{t} m a(X) m\right] .
$$

Recall the basis of $\mathfrak{g}$ from (23). The following formulas for $a(X)$ are easily verified: for any $s \in \mathbb{C}$,

$$
a(s E)=a(s H)=a(s e)=0, \quad a(s F)=-\tilde{z} s z, \quad a(s f)=2 s z .
$$


Some calculation using these formulas and the definition of the action of $G$ on $\mathbb{H}_{\mathcal{Q}} \times \mathcal{Q}$ yields the following formulas for all $s \in \mathbb{C}$ :

$$
\begin{array}{ccc}
\lambda_{m}(s E)= & -2 \operatorname{Re}\left(s \partial_{x}\right), \\
\lambda_{m}(s H)= & -2 \operatorname{Re}\left(s\left(2 x \partial_{x}+y \partial_{y}+u \partial_{u}+v \partial_{v}\right)\right), \\
\lambda_{m}(s F)= & 2 \operatorname{Re}\left(\left(s x^{2}-\bar{s} y^{2}\right) \partial_{x}+s x y \partial_{y}+(s x u-\bar{s} y \bar{v}) \partial_{u}\right. \\
& \left.\quad+(s x v+\bar{s} y \bar{u}) \partial_{v}\right)+2 \pi i \mathcal{T}_{\mathcal{R}}\left[{ }^{t} m \tilde{z} s z m\right], \\
\lambda_{m}(s e)= & -2 \operatorname{Re}\left(s \partial_{u}\right), \\
\lambda_{m}(s f)= & -2 \operatorname{Re}\left(s x \partial_{u}+\bar{s} y \partial_{v}\right)-8 \pi i \operatorname{Re}\left({ }^{t} m m s u\right)+8 \pi^{t} m \bar{m} \operatorname{Re}(s v) .
\end{array}
$$

From these formulas we deduce

$$
\begin{aligned}
\left.\lambda_{m}\left(Q_{\tau}\right)\right|_{(\mathbf{k}, 0)} & =\left.4\left(4 \partial_{x} \partial_{\bar{x}}+\partial_{y}^{2}-\partial_{y}\right)\right|_{(\mathbf{k}, 0)}, \\
\left.\lambda_{m}\left(Q_{z}\right)\right|_{(\mathbf{k}, 0)} & =\left.4\left(\partial_{u} \partial_{\bar{u}}+\partial_{v} \partial_{\bar{v}}\right)\right|_{(\mathbf{k}, 0)}-16 \pi^{t} m \bar{m} .
\end{aligned}
$$

In order to apply $(21)$, fix $\left(\tau_{0}, z_{0}\right):=\left(x_{0}+y_{0} \mathbf{k}, u_{0}+v_{0} \mathbf{k}\right)$ in $\mathbb{H}_{\mathcal{Q}} \times \mathcal{Q}$. Define $g_{0} \in G$ by

$$
g_{0}:=\left(M_{0}, X_{0}, 0\right), \quad M_{0}:=y_{0}^{-1 / 2}\left(\begin{array}{cc}
y_{0} & x_{0} \\
0 & 1
\end{array}\right), \quad X_{0}:=y_{0}^{-1 / 2}\left(\bar{v}_{0}, u_{0}\right) .
$$

Then

$$
g_{0} \circ(\tau, z)=\left(x_{0}+y_{0} \tau, u_{0}+\tau \bar{v}_{0}+y_{0}^{1 / 2} z\right) .
$$

In particular, $g_{0} \circ(\mathbf{k}, 0)=\left(\tau_{0}, z_{0}\right)$. Therefore $(21)$ becomes

$$
\begin{aligned}
& \left(\mathrm{IDO}_{m}(Q \bullet) f\right)\left(\tau_{0}, z_{0}\right) \\
& \quad=\left.\alpha_{m}^{-1}\left(g_{0},(\mathbf{k}, 0)\right) \lambda_{m}(Q \bullet)\right|_{(\tau, z)=(\mathbf{k}, 0)}\left[\alpha_{m}\left(g_{0},(\tau, z)\right) f\left(g_{0} \circ(\tau, z)\right)\right] .
\end{aligned}
$$

Recall that $\alpha_{m}=\exp 2 \pi i \mathcal{I}_{\mathcal{R}}\left[{ }^{t}\right.$ mam $]$. One computes

$$
a\left(g_{0},(\tau, z)\right)=\bar{v}_{0}\left(u_{0}+2 z y_{0}^{1 / 2}+\tau \bar{v}_{0}\right) / y_{0} .
$$

The factors contributed by the summand $\bar{v}_{0} u_{0} / y_{0}$ in this formula to the two $\alpha_{m}$ 's in (26) cancel, as they are constant in $(\tau, z)$. Thus we arrive at

$$
\begin{aligned}
&\left(\operatorname{IDO}_{m}\left(Q_{\bullet}\right) f\right)\left(\tau_{0}, z_{0}\right)= \exp \left\{-4 \pi i^{t} m \bar{m} v_{0} \bar{v}_{0} / y_{0}\right\} \\
& \times\left.\lambda_{m}(Q \bullet)\right|_{(\tau, z)=(\mathbf{k}, 0)}( \exp \left\{2 \pi i y_{0}^{-1} \mathcal{T}_{\mathcal{R}}\left[{ }^{t} m \bar{v}_{0}\left(2 z y_{0}^{1 / 2}+\tau \bar{v}_{0}\right) m\right]\right\} \\
&\left.\times f\left(x_{0}+y_{0} \tau, u_{0}+\tau \bar{v}_{0}+y_{0}^{1 / 2} z\right)\right) .
\end{aligned}
$$

From here a straightforward calculation finishes the proof. 
5.4. Remarks. Berndt and Schmidt [1] and Yang [24] have carried out calculations analogous to those of Section 5 for $\mathrm{SL}_{2} \mathbb{R} \ltimes\left(\mathbb{R}^{2} \tilde{\times} \mathbb{R}\right)$, the real Jacobi group. Here the algebra of invariant differential operators has two second order and two third order generators, all of which are given explicitly in both references, in [1] for all $k$ and $m$ and in [24] for $k=m=0$. The analogous calculations for $\mathrm{SL}_{2} \mathbb{C}$ are classical; see for example [4]. There the algebra of invariants is generated by the Laplace-Beltrami operator, which may be obtained from $D_{0,0}^{\tau}$ by deleting all terms involving $u$ or $v$.

We remark that in [1] the invariant operators are computed using an ingenious trick: they are factored as products of first order covariant operators between two different slash actions, whose $k$-values differ by 1 or 2 . To our knowledge this trick originated with Maass [17] for slash actions of $\mathrm{SL}_{2} \mathbb{R}$. It was also used by Friedberg [5] to compute the invariant operators on the vector-valued slash actions of $\mathrm{SL}_{2} \mathbb{C}$.

The real analog of the formula for $\alpha_{k, m}$ may be found in Eichler and Zagier [3], as well as in [1]. As we have mentioned, in the complex case $\alpha_{k, 0}$ is a coboundary. In fact this is obvious without calculation, as $\mathrm{SU}_{2}$ has no non-trivial characters. In the real case $\alpha_{k, 0}$ is not a coboundary but $k$ must be integral. The point is that there $S^{1}$ replaces $\mathrm{SU}_{2}$, and $S^{1}$ has non-trivial characters indexed by $\mathbb{Z}$. (A more down-toearth explanation of the reason that $k$ must be integral in the real case is that there $\left(M_{21} \tau+M_{22}\right)^{-k}$ replaces $\mathcal{N}^{-k}\left(M_{21} \tau+M_{22}\right)$ in the formula for $\left.\alpha_{k, m}.\right)$

It would be interesting to extend Friedberg's computation of the invariant differential operators on vector-valued slash actions of $\mathrm{SL}_{2} \mathbb{C}$ to compute the invariant differential operators on vector-valued slash actions of our group $G$. These slash actions arise from the higher dimensional irreducible representations of $\mathrm{SU}_{2} \times Z(G)$, and so they have a genuine parameter $k \in \mathbb{N}$. The trick of Maass mentioned above will reduce the calculations considerably.

There are two alternate systems of coordinates in which the formulas of Proposition 5.7 are simpler. First, one can use the following "quaternionic derivatives":

$$
\begin{aligned}
\partial_{\tau}:=\partial_{x}-\frac{1}{2} \mathbf{k} \partial_{y}, & \partial_{\bar{\tau}}:=\overline{\partial_{\tau}}=\partial_{\bar{x}}+\frac{1}{2} \mathbf{k} \partial_{y}, \\
\partial_{z}:=\partial_{u}-\mathbf{k} \partial_{v}, & \partial_{\bar{z}}:=\overline{\partial_{z}}=\partial_{\bar{u}}+\partial_{\bar{v}} \mathbf{k}=\partial_{\bar{u}}+\mathbf{k} \partial_{v} .
\end{aligned}
$$

These operators do not have all the nice properties of the complex partials, but we do get the following:

$$
\begin{aligned}
D_{k, m}^{z}= & y \partial_{z} \partial_{\bar{z}}+4 \pi i \mathcal{T}_{\mathcal{R}}\left[{ }^{t} m \bar{v} \partial_{\bar{z}} m\right]-16 \pi^{2}\left(\left({ }^{t} m \bar{m}\right)^{2}-\left|{ }^{t} m m\right|^{2}\right) v \bar{v} / y, \\
D_{k, m}^{\tau}= & 4 y^{2} \partial_{\tau} \partial_{\bar{\tau}}+(2 k-1) y \partial_{y}+8 y \operatorname{Re}\left(\partial_{\bar{\tau}} \bar{v} \partial_{z}\right) \\
& +4 v \bar{v} \partial_{z} \partial_{\bar{z}}-4 \operatorname{Im}^{2}\left(v \partial_{v}\right)+4(k-1) \operatorname{Re}\left(v \partial_{v}\right) \\
& +8 \pi i \mathcal{T}_{\mathcal{R}}\left[m \cdot \bar{v} \partial_{\bar{\tau}} \bar{v} m\right]+8 \pi i \frac{v \bar{v}}{y} \mathcal{T}_{\mathcal{R}}\left[m \cdot \bar{v} \partial_{\bar{z}} m\right] \\
& -16 \pi^{2}\left(\left({ }^{t} m \bar{m}\right)^{2}-\left|{ }^{t} m m\right|^{2}\right)(v \bar{v} / y)^{2}-4(2 k-1) \pi m \cdot \bar{m}(v \bar{v} / y) .
\end{aligned}
$$

Second, we can use "complex S-coordinates". The coordinates $(x, y, u, v)$ which we have been using are what would be called "complex EZ-coordinates" in [1]. Define $p, q \in \mathbb{C}$ by the equation $z:=\tau p+q$, so that $p=\bar{v} / y$ and $q=u-x \bar{v} / y$. The complex S-coordinates are $(x, y, p, q)$. The formula for $D_{k, m}^{\tau}$ in S-coordinates is much 
simpler, essentially because they correspond to writing the semidirect product group $G$ with $\mathrm{SL}_{2} \mathbb{C}$ on the right. We give only $D_{0, m}^{\tau}$; the formula for $D_{k, m}^{\tau}$ may be obtained from conjugation by $y^{-k}$. Writing $\partial^{S}$ for the $S$-coordinate partial derivatives (and continuing to use the quaternionic derivative $\partial_{\tau}$ ), we find

$$
\begin{aligned}
D_{0, m}^{\tau}= & 4 y^{2} \partial_{\tau}^{S} \partial_{\bar{\tau}}^{S}-y \partial_{y}^{S}+8 \pi i \mathcal{T}_{\mathcal{R}}\left[m \cdot p \partial \frac{S}{\bar{\tau}} p m\right] \\
& -16 \pi^{2}\left(\left({ }^{t} m \bar{m}\right)^{2}-\left|{ }^{t} m m\right|^{2}\right)(y p \bar{p})^{2}+4 \pi m \cdot \bar{m} y p \bar{p}
\end{aligned}
$$

In particular, $\partial_{p}^{S}$ and $\partial_{q}^{S}$ appear only in $D_{k, m}^{z}$. The formula for this operator is actually a little more complicated than before, so we omit it.

We conclude with a sharpening of Corollary 5.4, based on the observation that $\mathbb{D}_{\alpha}(G / K)$ is noncommutative. (It would be interesting to have an efficient method to compute relations as well as generators for $\mathbb{D}_{\alpha}(G / K)$. Computing the third order generators using the method of this paper would be very tedious, and it appears that computing the commutation relations between all the generators by hand would be prohibitively difficult.)

Proposition 5.8. For any cocycle $\alpha \in Z^{1}(G, K)$, the algebra $\mathbb{D}_{\alpha}(G / K)$ is generated by two second order and two third order operators.

Proof: Let $\mathcal{C}$ be the commutator $\left[\operatorname{IDO}_{\alpha}\left(Q_{\tau}\right), \operatorname{IDO}_{\alpha}\left(Q_{z}\right)\right]$. It will suffice to prove that $\mathcal{C}$ is a third order operator, as then it can be used to replace one of the three third order operators in Corollary 5.4 (we do not know which one). For this, use (21) to see that for $\Omega \in \mathcal{S}(\mathfrak{m})^{K}$, the symbol of $\operatorname{IDO}_{\alpha}(\Omega)$ is independent of $\alpha$. Therefore $\mathcal{C}$ is third order if and only if $\left[D_{k, m}^{\tau}, D_{k, m}^{z}\right]$ is third order, which is easy to verify because one need keep track only of the second order symbols of $D_{k, m}^{\tau}$ and $D_{k, m}^{z}$.

\section{References}

[1] Berndt, R., And Schmidt, R. Elements of the representation theory of the Jacobi group. Progr. Math. 163. Birkhäuser, Basel, 1998.

[2] Bump, D. Automorphic forms and representations, vol. 55 of Cambridge Studies in Advanced Mathematics. Cambridge University Press, 1997.

[3] Eichler, M., And Zagier, D. The theory of Jacobi forms. Birkhäuser, Boston, 1985.

[4] Elstrodt, J., Grunewald, F., And Mennicke, J. Groups acting on hyperbolic space. Springer Monographs in Mathematics. Springer, Berlin, 1998.

[5] Friedberg, S. Differential operators and theta series. Trans. Amer. Math. Soc. 287, no. 2 (1985), 569-589.

[6] Goodman, R., And Wallach, N. Representations and invariants of the classical groups. Cambridge University Press, 1998.

[7] Hecke, E. Über die L-Funktionen und den Dirichletschen Primzahlsatz für einen beliebigen Zahlkörper. Nachrichten der K. Gesellschaft der Wissenschaften zu Göttingen, Mathematischphysikalische Klasse (1917), 299-318.

[8] Heim, B. Analytic Jacobi Eisenstein series and the Shimura method. J. Math. Kyoto Univ. 43, no. 3 (2003), 451-464.

[9] Hejhal, D. The Selberg trace formula for $\operatorname{PSL}(2, R)$. I, vol. 548 of Lecture Notes in Math. Springer, 1976.

[10] Hejhal, D. The Selberg trace formula for $\operatorname{PSL}(2, R)$. II, vol. 1001 of Lecture Notes in Math. Springer, 1983.

[11] Helgason, S. Invariant differential operators and eigenspace representations. In Representation theory of Lie groups (1979), vol. 34 of London Math. Soc. Lecture Note Series, Cambridge University Press, pp. 236-286. 
[12] Imamō̄lu, Ö. The Kubota symbol for $\operatorname{Sp}(2 n, Q(i))$. J. Number Theory 52 (1995), 17-34.

[13] IwANIEC, H. Spectral methods of automorphic forms, vol. 53 of Graduate Studies in Mathematics. AMS, 2002.

[14] Iwaniec, H., And Kowalski, E. Analytic Number Theory, vol. 53 of American Mathematical Society Colloquium Publications. AMS, 2004.

[15] MaAss, H. Über eine neue Art von nichtanalytischen automorphen Funktionen und die Bestimmung Dirichletscher Reihen durch Funktionalgleichungen. Math. Ann. 121 (1949), 141-183.

[16] MaAss, H. Die Differentialgleichungen in der Theorie der Siegelschen Modulfunktionen. Math. Ann. 126 (1953), 44-68.

[17] MaAss, H. Lectures on modular forms of one complex variable. Tata Institute of Fundamental Research Lectures on Mathematics 29. Bombay, 1964.

[18] Richter, O. Theta functions of quadratic forms over imaginary quadratic fields. Acta Arith. 92, no. 1 (2000), 1-9.

[19] Richter, O., And Skogman, H. Jacobi theta functions over number fields. Monatsh. Math. 141, no. 3 (2004), 219-235.

[20] Shimura, G. On the holomorphicity of certain Dirichlet series. Proc. London Math. Soc. 31, no. 1 (1975), 79-98.

[21] Skogman, H. Jacobi forms over imaginary quadratic number fields. Acta Arith. 96, no. 4 (2001), 333-350.

[22] Stanley, R. Invariants of finite groups and their applications to combinatorics. Bull. Amer. Math. Soc. 1 (1979), 475-511.

[23] Terras, A. Harmonic analysis on symmetric spaces and applications. I. Springer, New York, 1985.

[24] YAng, J. A note on Maass-Jacobi forms. Kyungpook Math. J. 43, no. 4 (2003), 547-566.

[25] Ziegler, C. Jacobi forms of higher degree. Abh. Math. Sem. Univ. Hamburg, 59 (1989), 191224.

School of Mathematics, University of Minnesota, Minneapolis, MN 55455, U.S.A.

E-mail address: bringman@math.umn.edu

Department of Mathematics, University of North Texas, Denton, TX 76203, U.S.A.

E-mail address: conley@unt.edu

Department of Mathematics, University of North Texas, Denton, TX 76203, U.S.A.

E-mail address: richter@unt.edu 